\title{
Anti-cancer Immunotherapy Epitope-peptides Vaccination in Patients with Refractory/Persistent Disease of Cervical Cancer and Ovarian Cancer (Phase 1 Studies)
}

\author{
Satoshi Takeuchi ${ }^{1,5}{ }^{\text {, * }}$, Tadahiro Shoji ${ }^{1}$, Masahiro Kagabu ${ }^{1}$, Tatsuya Honda ${ }^{1}$, Tadayuki Nagasawa ${ }^{1}$, \\ Yukari Nitta $^{1}$, Toru Sugiyama ${ }^{1,2}$, Sachiko Yoshimura ${ }^{3}$, Yusuke Nakamura ${ }^{3,4}$ \\ ${ }^{1}$ Department of Obstetrics and Gynecology, Iwate Medical University School of Medicine Morioka, Iwate, Japan \\ ${ }^{2}$ Women's Cancer Center, Gynecologic Oncology, Takagi Hospital, International, University of Health and Welfare, Ookawa City, Fukuoka, \\ Japan \\ ${ }^{3}$ Department of Cancer, The Institute of Medical Science, The University of Tokyo, Tokyo, Japan \\ ${ }^{4}$ Cancer Institute Hospital of JFCR (Japanese Foundation for Cancer Research), Tokyo, Japan \\ ${ }^{5}$ Division of Gynecologic Oncology, Department of Gynecology, Women's Health Care, Kobe Tokushukai Hospital, Kobe, Hyogo, Japan
}

\section{Email address:}

s-take-imugo@zeus.eonet.ne.jp (S. Takeuchi),tshoji@iwate-med.ac.jp (T. Shoji),m.kagabu@nifty.com (M. Kagabu), tatsu-h@ninus.ocn.ne.jp (T. Honda), tnagasawa@iwate-med.ac.jp (T. Nagasawa),tprtf842@ybb.ne.jp (Y. Nitta), sugiyama@kouhoukai.org (T. Sugiyama),sc-yoshimura@umin.ac.jp (S. Yoshimura), yusuke.nakamura@jfcr.or.jp (Y. Nakamura)

${ }^{*}$ Corresponding author

\section{To cite this article:}

Satoshi Takeuchi, Tadahiro Shoji, Masahiro Kagabu, Tatsuya Honda, Tadayuki Nagasawa, Yukari Nitta, Toru Sugiyama, Sachiko Yoshimura, Yusuke Nakamura. Anti-cancer Immunotherapy Epitope-peptides Vaccination in Patients with Refractory/Persistent Disease of Cervical Cancer and Ovarian Cancer (Phase 1 Studies). Cancer Research Journal. Vol. 7, No. 3, 2019, pp. 106-116. doi: 10.11648/j.crj.20190703.15

Received: August 1, 2019; Accepted: September 11, 2019; Published: September 24, 2019

\begin{abstract}
Despite the improvement of treatments, refractory or chemotherapy resistant ovarian and cervical cancers have been still incurable. In such tumors, the actionable salvage gene-pathways of up-regulating lung cancer 10 (URLC10), hypoxia inducible factor (HIF) and its core protein HIG2- tumor growth factor beta (TGF beta)- the Caenorhabditis elegans SMA ("small" worm phenotype) and Drosophila Mothers Against Decapentaplegic (SMAD), maternal embryonic leucine zipper kinase (MELK)- forkhead box M1 (FOXM1) which induces and stimulates stathmin concerning cell (vascular endothelial cell and tumor cell) migration and counter pathway of P53, and holliday junction recognition protein (HJURP)-histone H3-like centromeric protein A (CEMPA)-Histone, which play important roles in tumor proliferation, metastasis and cell cycling. They had been shifted from original driver gene such as Ras-MAPK or PIK3CA-mTOR. Furthermore, tumor specific microenvironmental factors such as vascular endothelial growth factor (VEGF) receptors facilitate tumor new-angiogenesis, invasion and metastasis, as well. We found human leukocyte antigen (HLA)-A*2402 and 0201 restricted epitope neo-antigens or, epitope peptides of VEGF receptor 1 and 2, using micro-cDNA assay form clinical samples. The peptides consisted in nine to eleven mer peptides, which were presented by HLA (major histocompatibility 1) on cell membrane. We administered the multiple peptides subcutaneously as vaccination and it activated intrinsic cell immune system of cytotoxic T cell (CTL). We conducted a phase $1 / 2$ study of those peptides vaccine (PV) cocktails to elucidate their toxicity profiles and efficacy from 4 June 2010 to Jan 2013 for phase 1 studies, and subsequently continued phase 2 studies at outpatient's clinic of our hospital. PV were administered at a dose of $1 \mathrm{mg}$ of each peptide with MONTANIDE*ISA51 (SEPPIC Co. Ltd, France). Enrollees were obtained written informed consent after our IRB approval on 3 June 2010. In results, no major adverse events were seen except dermatologic reactions at injection site. One patient showed complete response, two showed partial response and 10 showed stable disease out of 22 evaluable patients. Median overall survival was 5 months and 9 months in HLA-A2402 and 0201 group, respectively. In conclusion, these findings suggest the peptides cocktail vaccines were safe and applicable for advanced/recurrent OC.
\end{abstract}


Keywords: Immunotherapy, Cancer Vaccination, Epitope Peptide, Ovarian Cancer, Cervical Cancer, HLA, Chemotherapy Resistance, Persistent Disease

\section{Introduction}

Immunotherapy has been the fourth promising treatment for advanced unresectable or heavily treated cancers, especially, for the patients with resistant to chemotherapy of multiple lines. Nowadays, the immune-checkpoint inhibitors like anti-CTLA4 (cytotoxic T lymphocyte-associated protein 4) [1], anti-PD-1 (programmed cell death 1) [2], or antiPDL-1 (programmed cell death-ligand 1) [3] antibodies showed excellent outcomes [4]. In our studies, the specific antigens restricted by human leukocyte antigen (HLA) had been harvested by genome wide association studies (GWAS) [5], and using them, the cancer therapeutic vaccination therapy had been reported in gastric cancer [6], lung cancer [5], pancreatic cancer [7], esophageal cancer [8], colorectal cancer [7], and head \& neck cancer [6].

In gynecologic cancers, ovarian cancer (OC) is most fatal (ACS 2018 estimated death rate: 63.3\%), cervical cancer (CC) $(31.5 \%)$ and uterine corpus cancer (18.0\%) follows [9]). This fact suggests that it remains very hard to achieve cure in advanced or recurrent $\mathrm{OC}$ and $\mathrm{CC}$, and it has been the problem in gynecologic oncology. Mainly, by Gynecologic Oncology Group (GOG) in United States, the standard treatment of combined chemo-regimen with taxane and platinum were identified $[10,11]$, and addition of molecular targeted agents, bevacizumab had been studied [12] in OC and in CC [13]. Newly emerging Poly (ADP-ribose) polymerase (PARP) inhibitors, olaparib [14] and niraparib or atezolizumab and so on, are promising and the candidate of agent which would like to cure the ovarian cancer.

In fact, the chemotherapy for OC had achieved better response rate nearly $90 \%$ and longer progression free survival (PFS) in patients with advanced OC, however, the recurrence rate has been still over $85 \%$ [10], and longer overall survival (OS) has not obtained yet in advanced stage disease.

Considering the recurrence of the tumor, it depends on residual tumor cells or probably, tumor stem cells which are usually chemo-resistant. Speaking of chemo-resistance, OCs have heterogeneity not only in sub-histologic types but also even if, in most frequent sub-type, such as high-grade serous carcinoma (HG-S), which was composed of various cyclingphasic cells from mitotic cells to resting or stem-like cell [15]. Thus, it is necessary that the new strategy for eradicating and eliminating of residual tumor cells should be explored. Actually, the immune therapy might be one of the methods for cure cancer because of ability to precise detection and accurate attack of cytotoxic T cell (CTL) with 'homing'. The strategy using specific CTL demands specific targets only expressed on tumor cell and not on normal cells of every organs. We had chosen the specific antigens restricted HLA-A loci which are the proliferative driver-gene products (peptides of 9 mers to 11 mers) of tumor cells and peptides from vascular endothelial growth factor receptor 1 (VEGFR1) and 2 (VEGFR2), which were taken from tumor associated vascular endothelial cells as targets from tumormicro environment.

We conducted phase $1(\mathrm{P} 1)$ and $2(\mathrm{P} 2)$ study for patients with persistent disease of heavily treated OC or CC using HLA restricted epitope-peptides as tumor specific neoantigens in multi-peptides cocktail vaccination (PV). For these patients of cohort, immunotherapy using activation of innate CTL pathway was presented.

The first six enrolled patients were P1 study in modified Fibonacci method. The significant points of this study were as follows:

Firstly, these had been phase $1 / 2$ studies of multiple peptides vaccine comprised of 2 to 5 peptides.

Secondly, as onco-antigens, new epitope-peptides of MELK (maternal embryonic leucine zipper kinase) had been used first in human. After confirmation of safety and efficacy, further patients were accrued in P2 trial (including P1).

The primary endpoint had been safety and secondary endpoints had been response, immunological evaluation using Enzyme-Linked Immuno-Spot (ELISpot) assay, and patient's outcome.

\section{Methods and Patients}

\subsection{Methods}

\subsubsection{Study Design and Endpoints}

Study design was prospective, interventional, nonrandomized exploratory phase 1 and 2 trial of one arm. P1 part was performed according to modified Fibonacci method in one dose level. Dose limiting factors were grade 4 in hematologic toxicity lasting more than 7 days, and grade 3 or more in nonhematologic toxicities without dermatologic reactions. The adverse event (AE) was evaluated by National Cancer Institution-Common Toxicity Criteria of Adverse Events version. 3.0 (NCI-CTCAE ver.3.0). As for response, the response rate (RR) which estimated by immune-response Response Evaluation Criteria in Solid Tumor version 1.1 (irRECIST v.1.1). Overall survival (OS) in phase 2 and immunological evaluation of CTL responses by ELISpot assay of peripheral blood mononuclear cells had been assessed. In ethically, ethical institutional review board had approved these studies and all patients were enrolled after obtaining of written informed consent using approved documents. The ERB was registered in US OHRP (OHPR Federal wide Assurance Number : FWA0010884 as Iwate Medical University IRB\#1) and the approved numbers of these study wereH22-46, 47, 48 and 49 on June $3^{\text {rd }}, 2011$ ). 
These studies had registered in UMIN as UMIN 000003860 , $000003862,000003902,000003903$.

\subsubsection{Study Period and Location}

Accrual initiated on 2010/ 6/4 (after approval by ERB). Study closure was 2016/3/31 in CC and 2017/7/10 in OC, and observation had been continued until patient's death or 1 year after of the administration of last accrued patient which was 2018/8/10 The protocol treatment (intervention) was discontinued on 2017/7/10. As for phase 1, the last phase 1 of CC0205 on 12 April 2012. And study location was our outpatients' clinic (department of obstetrics and gynecology, Iwate Medical University Hospital: 19-1 Uchimaru Morioka Iwate Japan 0208505

\subsection{Participants}

\subsubsection{Patient's Eligibility and Exclusion}

(i) Patient's eligibility

Patients were histologically confirmed $\mathrm{CC}$ or epithelial OC with recurrent, refractory and/or persistent disease. The standard treatment, such as surgery, radiotherapy, chemotherapy, or concurrent chemoradiotherapy (CCRT) must have been performed as prior treatment. As for OC, patient had received more than three lines of chemoregimens after standard treatment, otherwise allergy to some agents. In CC, more than one adjuvant/salvage chemotherapy must have been performed. Some patients who insist on refusal strongly to have further lines of chemotherapy after first line were allowed to be enrolled from the ethical point of view, as well. Patients who would be able to be alive more than 12 weeks at baseline, and they had been received fully prior treatments and the interval of 28 days had been passed at baseline. Concomitant chemotherapy, radiotherapy or other immune therapy, without surgery alone, was not allowed. Patients who had announced best supportive care (BSC) or no further chemotherapy or radiotherapy were eligible bur steroid use was not allowed. All patients must be performance status (PS) 0-2 by ECOG (Eastern Cooperative Oncology Group). Age limitation was over 20 up to less than 80 , considering the preserved function of bone marrow and adequate intrinsic immunity. As for HLA*A subtypes, HLA*A-0201, or HLA*A-2402 must be necessary. Patients who had had active other cancers or past experience of cancers and who had had brain metastasis of cancer (even post treatment) were not allowed to include these studies.

(ii) Exclusion criteria

Patients who have conflicted eligibility criteria. Patients who had had been depressed in immunity such as HIV were excluded. Patient who could not come outpatient clinic by herself or cognitive insufficiency were excluded. Patient who were determined as inappropriate by investigators were excluded.

(iii) Studies' constitution

Studies were divided to four groups according to HLA*A subtyping and disease, that is, HLA-A*0201 positive uterine CC (CC02), HLA-A*2402 positive CC (CC24), HLA$\mathrm{A}^{*} 0201$ positive OC $(\mathrm{OC} 02)$ and HLA-A*2402 positive OC
(OC24). The HLA-A*2402 was demonstrated $60 \%$ of Japanese and the HLA-A*0201 covers the rest $20 \%$. By use of them, $80 \%$ of Japanese women were speculated to be covered.

\subsubsection{Protocol Treatment}

Vaccination using tumor antigens were performed subcutaneous injection according to schedule.

(i) Tumor specific epitope peptides' constitution

Nakamura et al. identified various tumor-specific epitopepeptides by GWAS using cDNA microarray technology coupled with laser microdissection, they found novel HLA$A * 2402$ and HLA-A*0201 restricted epitope peptides of several genes related to salvage actionable proliferative pathways $[1,7,16-20]$.

Adding to the tumor cell attack using specific antigens stated above, we aimed dual attack in shutting the blood supply of tumor by destruction of microenvironment of tumor, using specific epitope-peptides antigens restricted $A * 0201$ and $A * 2402$.

(a) HLA-A*0201 CC patients: CC02

For cervical cancer (CC) (two peptides cocktail): $\mathrm{CC} 02$ cocktail consists of epitope peptides from URLC10 and HIG2

epURLC10: URLC10 (up-regulating lung cancer 10) [20] is a glycoprotein which was identified as neo-antigen with highly expression in non-small lung cancer (NCLC). Histopathologically, the positive rates of expression of ULRC10 by immune-histochemistry (ICH) were $90 \%$ in NSCLC, and $64 \%$ in uterine cervical squamous cell cancer, respectively [21]. $A^{*} 0201$ restricted peptides (LLLASIAAGL) from URLC10 showed good immunogenicity to CD8 positive T cell and induced CTL clone which has cytotoxic function [5]. In this trial HLA-A*0201 epitope peptides epURLC10A*0201-10-211 (LLLASIAAGL) was used.

epHIG2: HIG2 (hypoxia-inducible protein2) is a gene protein detected as specific tumor antigen highly expressed in clear cell carcinoma of kidney. It has not expressed in normal organs, and highly expressed in OC and CC. as well, such as $83 \%$ and $100 \%$ by IHC staining [16, 18], respectively. In this trial, $A^{*} 0201$-restricted epitope-peptides epHIG2-A*0201-9-4 (VLNLYLLGV) was used.

(b) HLA-A*0201-positive OC patients: OC02

For $A^{*} 0201$ positive OC, epHIG2, epVEGFR1 and epVEGFR2 were used.

epHIG: HIG2-A02-9-4 (VLNLYLLGV) (the same with epHIG peptide used in $\mathrm{CC} 02$ )

epVEGFR1: VEGFR1 comprises of 1338 amino acids and membrane protein which is essential to proliferation of angiogenesis of solid tumor located in the vascular endothelium membrane. VEGFR1 has immunogenicity as well, and it is applicable for new targeted antigen by which CTL can be induced and that detect and suppress the proliferation of the tumor cells [17]. In this trial, epVEGFR1-A*0201-9-770 (TLFWLLLTL) was used in these trials.

epVEGFR2: VEGFR2 comprises of 1356 amino acids and 
also the membrane protein which is essential to proliferation and migration of endothelium that induced angiogenesis of solid tumor, alone. VEGFR2, as well, has immunogenicity and it is the other targeted epitope peptide being applicable for vaccine therapy to cancer by the activated CTL. In this trial, epVEGFR2-A*0201-9-773 (VIAMFFWLL) was used [18].

(c) HLA-A*2402-positive CC patients: CC24

For CC with positivity for $A * 2402$, epitope peptides of epFOXM1, epMELK and epHJURP, were used.

epFOXM1 (forkhead box M1): FOXM1 is the proliferation regulator of $\mathrm{OC}, \mathrm{CC}$ and bladder cancer. It is known that the highly expression in lung cancer and was identified in protein located in nucleus comprises of 801 amino acids. In normal tissue, it shows highly expression in testes. In NSLC, it was expressed $86 \%$ of clinical samples and in $\mathrm{CC}$ and $\mathrm{OC}$, the positive rates accounted for $84 \%$ and $67 \%$, respectively [21]. Peptides of (IYTWIEDHF) was identified the immunogenicity and CTL clone was established. In this trial for $A^{*} 2402$ positive CC, A*2402 restricted epitope peptides of FOXM1-A*2402-9-262 (IYTWIEDHF) was used.

epMELK (maternal embryonic leucine zipper kinase): MELK is a protein composed of 651 amino acids, which is an expressed only testis in normal tissue. The rates of positivity for MELK in Clinical samples by immunohistochemistry were $76 \%$ in breast cancer, $93 \%$ in $\mathrm{CC}$, and $33 \%$ in OC [19], respectively. We identified the HLA*A2402 restricted MELK (EYCPGGNLF) with immunogenicity for CD8+Tcells and it induced the CTL clone. This CTL attacked the intrinsic generated MELK and A*2402 tumor cells. In this trial, epMELK-A*2402-9-87_7N (EYCPGGNLF) was used.

epHJURP (holliday junction recognition protein): HJURP was identified the new gene highly expressed in NCLC and comprises of 748 amino acids. Clinical specimen showed $100 \%$ positive $(12 / 12)$ in NCLC, and $89 \%(8 / 9)$ positive in $\mathrm{CC}$ and $85 \%$ [22] in OC. In this trial epHJURP $\mathrm{A}^{*} 2402$ restricted-9-408 (KWLISPVKI) was used.

(d) HLA-A*2402 OC patients: OC24

For positive patients (OC24), epitope peptides of epFOXM1, epMELK, epHJURP, epVEGFR1 and epVEGFR2 were used.

Those tumor specific epitope-peptides used were the same with epitope peptides used in CC24 stated above with restriction to HLA-A*2402.

All peptides cocktail sequences were shown in table 1 .

Table 1. Peptides and targets gene expression positivity in clinical samples.

\begin{tabular}{|c|c|c|c|}
\hline \multirow{2}{*}{ Target gene } & \multirow{2}{*}{ Peptides } & Positivity IHC \% & Positivity IHC \% \\
\hline & & Cervical Cancer & Ovarian Cancer \\
\hline \multicolumn{4}{|l|}{ HLA-A $* 201$} \\
\hline URLC10 -A*0201-10-211 & LLLASIAAGL & 94 & N/A \\
\hline HIG2-A*0201-9-4 & VLNLYLLGV & 100 & 83 \\
\hline VEGFR1-A*0201-9-770 & TLFWLLLTL & N/A & N/A \\
\hline VEGFR2-A*0201-9-773 & VIAMFFWLL & N/A & N/A \\
\hline \multicolumn{4}{|l|}{ HLA-A $* 2402$} \\
\hline FOXM1-A*2402-9-262 & IYTWIEDHF & 84 & 67 \\
\hline MELK-A*2402-9-87-7N & EYCPGGNLF & 93 & 33 \\
\hline HJURP-A*2402-9-408 & KWLISPVKI & 89 & 85 \\
\hline VEGFR1-A*2402-9-1084 & SYGVLLWEI & N/A & N/A \\
\hline VEGFR2-A*2402-9-169 & REVPDGNRI & N/A & N/A \\
\hline
\end{tabular}

Abbreviation: HLA (human leukocyte antigen), IHC (immuno-histochemical staining), URLC10 (up-regulation lung cancer 10), HIG2 (hypoxia inducible protein 2), VEGFR1 (vascular endothelial growth factor receptor 1), VEGFR2 (vascular endothelial growth factor receptor 2), FOXM1 (Forkhead box M1), MELK (maternal embryonic leucine zipper kinase), HJURP (holliday junction recognition protein)

(ii). Administration methods of Vaccine
Vaccination was performed by subcutaneous injection at left axillary region or right axillary region. If could not, inguinal regions were allowed as well.

Vaccination schedule was first 12 consecutive subcutaneous weekly injections at axillary regions followed additional 8 administrations (adm) in two- week intervals up to $20 \mathrm{adm}$, after that the interval was every 4 weeks (21-28 adm) and 3 months up to progression of the Figure 1. 


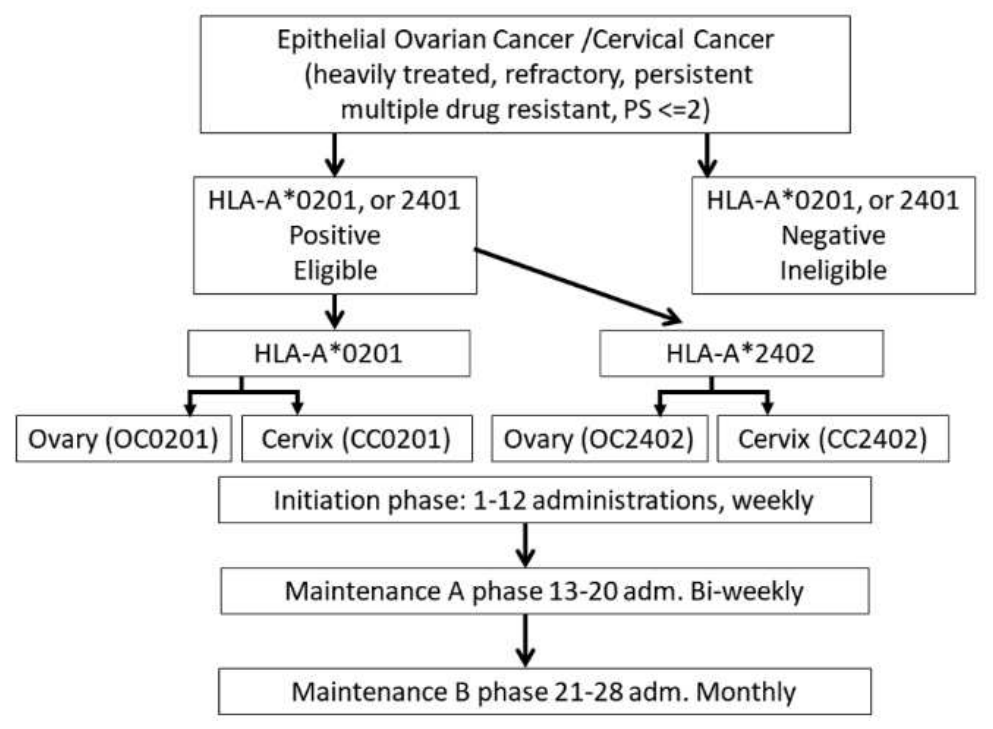

Figure 1. Schema of the protocol treatment. Informed consent was performed for HLA-A*0201 positive or *A2402 positive patients of this heavily treated cohort. Administration was performed in outpatient clinic, at first as initiation phase, weekly via subcutaneous injection for 12 weeks. Maintenance phase comprises of A phase (bi-weekly for 8 administrations, up-to 20 times in total) and B phase (monthly administration). Abbreviations; PS: performance status by ECOG, OC: $O C, C C: C C$, adm.: administration.

(iii) Evaluation of adverse events (toxicities) and response (a) Assessment of Toxicity

Safety was evaluated in all patients having received at least one administration of the vaccine and re-visited by assessment of adverse events (AEs). All patients were followed until recovery from toxicity or progressive of the disease. Normal organ functions and serum enzymes levels were checked except liver metastases case (up to three times of normal limits were allowed in AST and ALT). AEs were assessed in every visit and the worst grade of AEs were evaluated after 1 st course (after 4 administrations). The dose limiting toxicity (DLT) was defined as G4 hematologic toxicities lasting more than 1 week, and grade 3 or over of non-hematological toxicities and of unknown toxicity.

(b) Assessment of Response

To evaluate tumor response every patient must be performed imaging analysis (CT, MRI, PET-CT) within 28 days before enrollment as baseline (BL) then images were taken every after 2courses (every after 8 adm). Clinical responses were evaluated every 8 administrations by RECIST v. 1.1 and re-assessed by ir-RECIST by the same modality used at BL.

(c) Immunological response: Measurement of CTL responses using ELISpot assay

An enzyme-linked immune-spot (ELISpot) assay was performed to measure the peptide specific CTL response, as described previously [23]. For the evaluation of CTL, blood samples were obtained from the patients at the prevaccination period and after the 4, 8, 12 and 16 adm. Briefly, peripheral blood

mononuclear cells (PBMC) of blood samples were cultured with respective peptide and IL-2 (Novartis, Emeryville, CA) at $37^{\circ} \mathrm{C}$ for two weeks. Peptide was added into the culture at day 0 and day 7 . Following CD4+ cell depletion by Dynal CD4 positive isolation kit (Invitrogen,
Carlsbad, CA), IFN- $\gamma$ ELISpot assay was performed using Human IFN- $\gamma$ ELISPOT PLUS kit (MabTech, Nacka Strand, Sweden) according to the manufacturer's instructions. The number of peptide specific spots was calculated by subtracting the spot number in the control well from the spot number of wells with peptide pulsed TISI cells. The positivity of the antigen-specific $\mathrm{T}$ cell responses was classified into four grades $(-,+,++$ and +++$)$, depending on the peptide-specific spots at different responder/stimulator ratios. When the algorithm indicated,+++ or +++ , we judged it to be a positive case.

\section{Results}

\subsection{Patient's Characteristics}

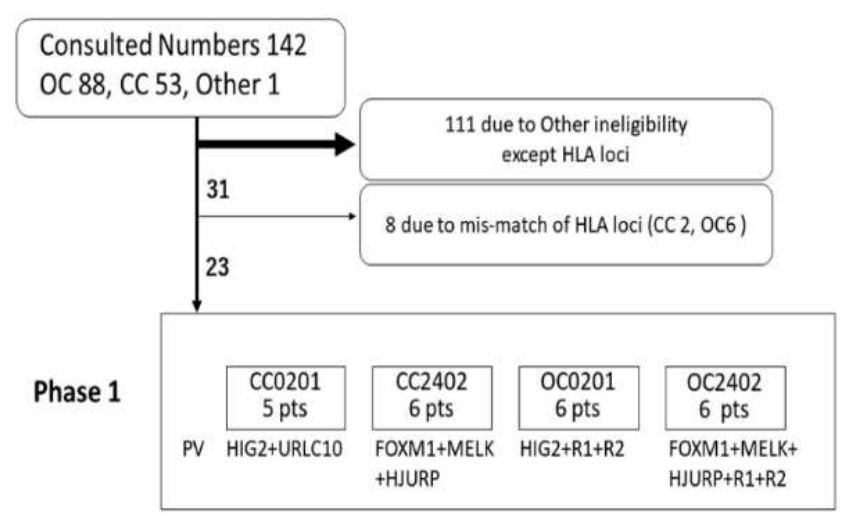

Figure 2. Diagram of patients' accrual and eligible patient number in phase 1 .

One hundred and forty-two patients inquired to us about our vaccination therapy. Participants who matched the eligibility criteria and did not conflict ineligible criteria were 31 patients. Twenty-three patients $(74.1 \%)$ out of 31 patients were suitable for HLA-A*0201 and/or A*2402 positive. and 
enrolled these trials were 23 in P1.

Abbreviations: cervical cancer (CC), ovarian cancer (OC).

As shown in Figure 2, the total number of patients referring consultation to join these clinical studies was 142 (88 OC, $53 \mathrm{CC}, 1$ Primary peritoneal cancer) and had reached the satisfied numbers in each trial. Patients of $\mathrm{CC}$ with HLA-A*0201 were five but all patients did not show DLT and no further eligible patient was enrolled during trial period. The distribution subtypes of histology were shown in Table 2 .

Table 2. Distribution of histologic types.

\begin{tabular}{llll}
\hline disease & subtype & HLA-A*0201 & HLA-A*2402 \\
\hline & serous & 4 & 4 \\
Ovarian & musinous & 0 & 0 \\
cancer & endometrioid & 1 & 1 \\
& clear cell & 1 & 1 \\
Cervical & SCC & 3 & 1 \\
cancer & ADC & 2 & 5 (1GAS, 1ADSQ) \\
\hline
\end{tabular}

Abbreviations: serous, serous carcinoma, mucinous, mucinous carcinoma: endometrioid, endometrioid carcinoma, clear cell, clear cell carcinoma: SCC, squamous cell carcinoma: ADC, adenocarcinoma: GAS, gastric type adenocarcinoma: ADSQ, adenosquamous cell carcinoma

The prior treatment demonstrated in table3. Both OC and
CC patients had been heavily treated. Molecular targeted agents were bevacizumab, MORAb 202 (humanized antihuman folate receptor alpha (FRA) antibody farletuzumab). The immunotherapy, such as alpha-beta dendritic cell therapy was performed in one patient in OC and CC.

Table 3. Prior treatments.

\begin{tabular}{lll}
\hline & OC (12) & CC (11) \\
\hline Prior CT & $2-11$ & $3-5(3)$ \\
(Max-Min) & (median 4) & $4 / 11$ \\
Prior RT & $3 / 12$ & $3 / 11$ \\
CCRT & $2 / 12$ & $1 / 11$ \\
Molecular agents & $2 / 12$ & $1 / 11$ \\
Immuno-therapy & $1 / 12$ & $0 / 11$ \\
Hormone therapy & $1 / 12$ & \\
\hline
\end{tabular}

Abbreviations: CCRT, concurrent chemoradiotherapy

\subsection{Adverse Events}

\section{Toxicity Profiles}

As for toxicity, no hematologic adverse events related to these agents were detected and non-hematologic toxicities profiles were shown in Table 4. Dermatologic reactions such as redness, swelling, induration, pain, itching under grade 2 at injection site were detected in most of patients.

Table 4. Hematologic and Non-hematologic toxicity profiles.

\begin{tabular}{|c|c|c|c|c|c|c|c|c|c|c|c|c|c|c|c|c|c|c|c|c|}
\hline \multirow{2}{*}{$\begin{array}{l}\text { NCI-CTC ver.3.0 } \\
\text { Grade }(G)\end{array}$} & \multicolumn{5}{|c|}{ CC0201 } & \multicolumn{5}{|c|}{ OC0201 } & \multicolumn{5}{|c|}{ CC2402 } & \multicolumn{5}{|c|}{ OC2402 } \\
\hline & G0 & G1 & G2 & G3 & G4 & G0 & G1 & G2 & G3 & G4 & G0 & G1 & G2 & G3 & G4 & G0 & G1 & G2 & G3 & G4 \\
\hline $\mathrm{HB}$ & 5 & 0 & 0 & 0 & 0 & 6 & 0 & 0 & 0 & 0 & 6 & 0 & 0 & 0 & 0 & 6 & 0 & 0 & 0 & 0 \\
\hline WBC & 4 & 1 & 0 & 0 & 0 & 6 & 0 & 0 & 0 & 0 & 6 & 0 & 0 & 0 & 0 & 6 & 0 & 0 & 0 & 0 \\
\hline ANC & 5 & 0 & 0 & 0 & 0 & 6 & 0 & 0 & 0 & 0 & 6 & 0 & 0 & 0 & 0 & 6 & 0 & 0 & 0 & 0 \\
\hline LYM & 3 & 0 & 2 & 0 & 0 & 2 & 0 & 4 & 0 & 0 & 1 & 0 & 2 & 3 & 0 & 3 & 0 & 1 & 0 & 2 \\
\hline PLT & 5 & 0 & 0 & 0 & 0 & 6 & 0 & 0 & 0 & 0 & 6 & 0 & 0 & 0 & 0 & 6 & 0 & 0 & 0 & 0 \\
\hline $\begin{array}{l}\text { Gastrointestinal } \\
\text { events }\end{array}$ & 5 & 0 & 0 & 0 & 0 & 6 & 0 & 0 & 0 & 0 & 6 & 0 & 0 & 0 & 0 & 6 & 0 & 0 & 0 & 0 \\
\hline Abdominal Pain & 5 & 0 & 0 & 0 & 0 & 6 & 0 & 0 & 0 & 0 & 6 & 0 & 0 & 0 & 0 & 6 & 0 & 0 & 0 & 0 \\
\hline Diarrhea & 5 & 0 & 0 & 0 & 0 & 6 & 0 & 0 & 0 & 0 & 6 & 0 & 0 & 0 & 0 & 6 & 0 & 0 & 0 & 0 \\
\hline Nausea & 5 & 0 & 0 & 0 & 0 & 6 & 0 & 0 & 0 & 0 & 6 & 0 & 0 & 0 & 0 & 6 & 0 & 0 & 0 & 0 \\
\hline Cardiac Disorder & 5 & 0 & 0 & 0 & 0 & 6 & 0 & 0 & 0 & 0 & 6 & 0 & 0 & 0 & 0 & 6 & 0 & 0 & 0 & 0 \\
\hline Asthenia & 5 & 0 & 0 & 0 & 0 & 6 & 0 & 0 & 0 & 0 & 6 & 0 & 0 & 0 & 0 & 6 & 0 & 0 & 0 & 0 \\
\hline Pneumonitis & 5 & 0 & 0 & 0 & 0 & 6 & 0 & 0 & 0 & 0 & 6 & 0 & 0 & 0 & 0 & 5 & 1 & 0 & 0 & 0 \\
\hline Infection & 5 & 0 & 0 & 0 & 0 & 6 & 0 & 0 & 0 & 0 & 6 & 0 & 0 & 0 & 0 & 6 & 0 & 0 & 0 & 0 \\
\hline $\begin{array}{l}\text { Hepatic } \\
\text { dysfunction }\end{array}$ & 5 & 0 & 0 & 0 & 0 & 6 & 0 & 0 & 0 & 0 & 6 & 0 & 0 & 0 & 0 & 6 & 0 & 0 & 0 & 0 \\
\hline Renal dysfunction & 5 & 0 & 0 & 0 & 0 & 6 & 0 & 0 & 0 & 0 & 6 & 0 & 0 & 0 & 0 & 6 & 0 & 0 & 0 & 0 \\
\hline Ileus & 5 & 0 & 0 & 0 & 0 & 5 & 1 & 0 & 0 & 0 & 6 & 0 & 0 & 0 & 0 & 6 & 0 & 0 & 0 & 0 \\
\hline Hypersensitivity & 5 & 0 & 0 & 0 & 0 & 6 & 0 & 0 & 0 & 0 & 6 & 0 & 0 & 0 & 0 & 6 & 0 & 0 & 0 & 0 \\
\hline $\begin{array}{l}\text { Dermatologic } \\
\text { Reactions }\end{array}$ & 1 & 4 & 0 & 0 & 0 & 1 & 4 & 1 & 0 & 0 & 0 & 6 & 0 & 0 & 0 & 3 & 3 & 0 & 0 & 0 \\
\hline General Fatigue & 5 & 0 & 0 & 0 & 0 & 6 & 0 & 0 & 0 & 0 & 6 & 0 & 0 & 0 & 0 & 4 & 2 & 0 & 0 & 0 \\
\hline Hypertension & 5 & 0 & 0 & 0 & 0 & 6 & 0 & 0 & 0 & 0 & 6 & 0 & 0 & 0 & 0 & 6 & 0 & 0 & 0 & 0 \\
\hline Protein Urine & 5 & 0 & 0 & 0 & 0 & 5 & 1 & 0 & 0 & 0 & 6 & 0 & 0 & 0 & 0 & 5 & 1 & 0 & 0 & 0 \\
\hline
\end{tabular}

\subsection{Response}

\subsubsection{Tumor Size (ir-RECIST Version 1.1)}

The responses were shown in figures 3 and 4 . Figure 3 showed sequential tumor response using ir-RECIST by spider plots. In order to assess the response by ir-RECIST imaging analyses were scheduled in same modality and same parameters, and most of the modality was CE-CT $(<5 \mathrm{~mm}$ slices: usually $3 \mathrm{~mm}$ ). The total sum of the target (TGT) lesions was followed up during the treatment. At this imaging diagnosis, new lesion or worsening/improvement of non-target (N-TGT) lesions were noted in some cases. The best response means frankly the sum of the longest length of TGT. The interval of imaging was every 8 administrations ( 8 weeks to 32 weeks). Some longer survivors were detected (Figure 2). 

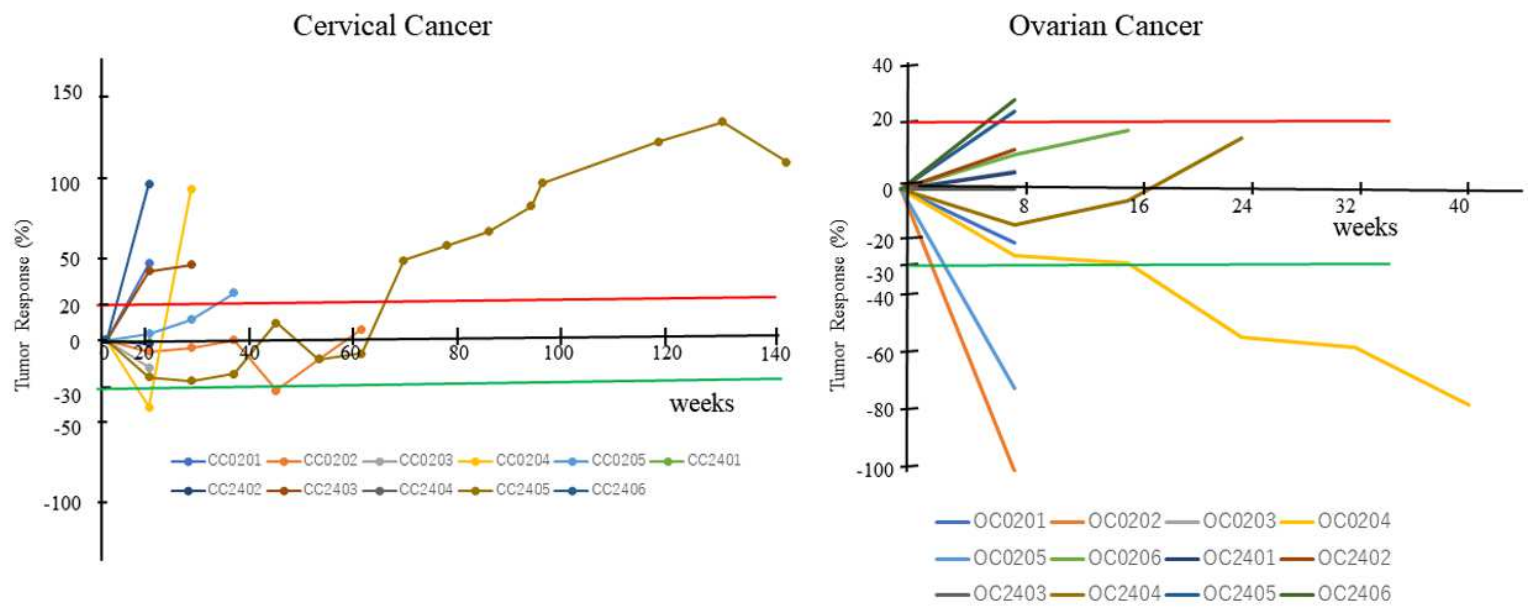

Figure 3. Tumor response of therapy. In order to assess the response by ir-RECIST imaging analyses were scheduled in same modality and same parameters (most of the modality was CE-CT ( $<5 \mathrm{~mm}$ slices: usually $3 \mathrm{~mm})$. The total sum of the target (TGT) lesions was followed.
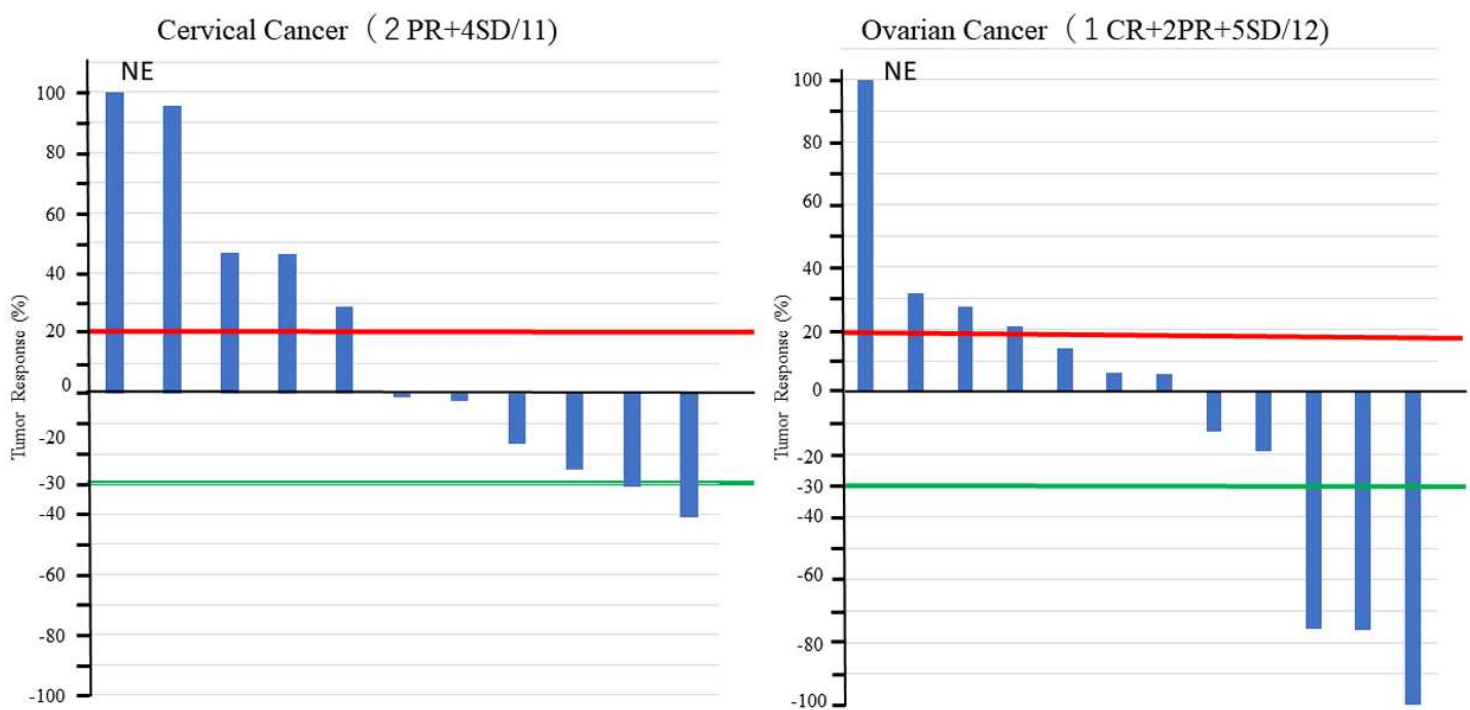

Figure 4. Best response (frank sum of the longest length of targets by ir-RECIST version 1.1). One patient who was not evaluable (NE) was seen in each cervical and ovarian group, who had received only baseline imaging. Red line shows $+20 \%$ increase (PD criteria) and green line shows $30 \%$ decrease (PR criteria). The bars between red and green lines equals to stable disease (SD).

As shown in Figure 4, in total 23 cases, 1 complete response (CR), 4 partial response (PR) and 9 stable disease (SD) were identified. Response rate (RR) was $21.7 \%$, and disease control rate (DCR) was $60.9 \%(14 / 23)$.

\subsubsection{Immunological Response}

Twenty two out of 23 patients could be analyzed the immunological response (Table 5). One in evaluable patient had not taken sample after administration. Only one patient out of 22 evaluable patients showed no immunological response by ELISpot assay, but she had dermatologic reactions. At baseline status, $23 \%(5 / 22)$ of patients had shown positive for new antigens, already. As for peptides of VEGF receptors (epVEGFR1 and R2), 34\% (4/12) of patients had had positivity for epVEGFR1 but none for epVEGFR2. After vaccination, the positive rate in new tumor antigens were $50 \%$ for epURLC10, $82 \%$ for epHIG2, $100 \%$ for epFOXM $1,100 \%$ for epMELK, $17 \%$ for epHJURP, $73 \%$ for epVEGFR 1 , and $64 \%$ for epVEGFR2, respectively.

Table 5. Immunological response analyses by ELISPOT assay (intensity of positivity).

\begin{tabular}{|c|c|c|c|c|c|c|c|c|c|c|c|c|c|c|}
\hline \multirow{2}{*}{ case } & \multicolumn{2}{|c|}{ epURLC10 } & \multicolumn{2}{|c|}{ еpHIG2 } & \multicolumn{2}{|c|}{ ерFOXM 1} & \multicolumn{2}{|c|}{ epMELK } & \multicolumn{2}{|c|}{ epHJURP } & \multicolumn{2}{|c|}{ epVEGFR1 } & \multicolumn{2}{|c|}{ epVEGFR2 } \\
\hline & BL & Post & BL & Post & BL & Post & BL & Post & BL & Post & BL & Post & BL & Post \\
\hline $\mathrm{CC} 0201$ & 0 & 0 & 0 & 0 & & & & & & & & & & \\
\hline CC0202 & 0 & 1 & 0 & 3 & & & & & & & & & & \\
\hline $\mathrm{CC} 0203$ & 0 & 0 & 0 & 3 & & & & & & & & & & \\
\hline CC0204 & 0 & 1 & 0 & 2 & & & & & & & & & & \\
\hline CC0205 & 0 & 0 & 0 & 3 & & & & & & & & & & \\
\hline
\end{tabular}




\begin{tabular}{|c|c|c|c|c|c|c|c|c|c|c|c|c|c|c|}
\hline \multirow{2}{*}{ case } & \multicolumn{2}{|c|}{ epURLC10 } & \multicolumn{2}{|c|}{ epHIG2 } & \multicolumn{2}{|c|}{ epFOXM 1} & \multicolumn{2}{|c|}{ epMELK } & \multicolumn{2}{|c|}{ epHJURP } & \multicolumn{2}{|c|}{ epVEGFR1 } & \multicolumn{2}{|c|}{ epVEGFR2 } \\
\hline & BL & Post & BL & Post & BL & Post & BL & Post & BL & Post & BL & Post & BL & Post \\
\hline CC2401 & & & & & 0 & 3 & 3 & 3 & 1 & 0 & & & & \\
\hline CC2402 & & & & & 0 & 3 & 1 & 1 & 2 & 0 & & & & \\
\hline CC2403 & & & & & NT & NT & 0 & 1 & 0 & 0 & & & & \\
\hline CC2404 & & & & & 0 & 3 & 0 & 3 & 0 & 0 & & & & \\
\hline $\mathrm{CC} 0205$ & & & & & 0 & 3 & 1 & 3 & 0 & 2 & & & & \\
\hline CC02026 & & & & & 0 & 3 & 0 & 3 & 0 & 0 & & & & \\
\hline OC0201 & & & 3 & 3 & & & & & & & 1 & 3 & 0 & 0 \\
\hline OC0202 & & & 0 & 3 & & & & & & & 2 & 3 & 0 & 1 \\
\hline OC0203 & & & 0 & 2 & & & & & & & 1 & 3 & 0 & 1 \\
\hline OC0204 & & & 0 & 3 & & & & & & & 0 & 3 & 0 & 1 \\
\hline OC0205 & & & 0 & 3 & & & & & & & & 3 & 0 & 2 \\
\hline OC0206 & & & 0 & 0 & & & & & & & 2 & 1 & 0 & 1 \\
\hline OC2401 & & & & & 0 & 3 & 0 & 3 & 0 & 0 & 0 & 0 & 0 & 0 \\
\hline OC2402 & & & & & 0 & 3 & 0 & 3 & 0 & 1 & 0 & 1 & 0 & 1 \\
\hline OC2403 & & & & & NT & NT & NT & NT & NT & NT & NT & NT & NT & NT \\
\hline OC2404 & & & & & 0 & 2 & 0 & 2 & 0 & 0 & 0 & 2 & 0 & 1 \\
\hline OC2405 & & & & & 1 & 3 & 1 & 3 & 1 & 0 & 0 & 0 & 0 & 0 \\
\hline OC2406 & & & & & 0 & 3 & 0 & 3 & 2 & 1 & 0 & 0 & 0 & 0 \\
\hline
\end{tabular}

Abbreviations: BL, baseline: Post, post vaccination, NT: not tested due to delinquency of samples

\section{Discussion}

The elucidation of several driver gene mutations in cancers, various actionable molecular targets in $\mathrm{OC}$ and $\mathrm{CC}$ were identified as well, and targeted agents were developed. In contrast, immunotherapy including our vaccination therapy according to HLA-A*typing is ultimate precision medicine in treatment of individuals as it were 'Homing Missile' unlike the 'Carpet Bombing' using treatment cytotoxic agents or immune-checkpoint inhibitors. In our studies, specific tumor antigens (epitope peptides) restricted HLA-*A2402 and 0201 were harvested by GWAS, we harvested nearly 30,000 peptides from transcriptome expressed in cervical and OC tumor cells and its tumor evoked micro-environment of the tumor specific endothelial cells.

Our peptides could cover $80 \%$ of Japanese women as stated above and would be appropriate for these vaccine trials. HLA-A*typing was organized as biomarker in these studies. Multi-cocktails of peptides would achieve completely eliminate tumor cells in a theoretical manner. Our selection of new onco-antigens was very reasonable.

URLC10: It was a core gene of proliferative pathway in squamous cell carcinoma.

HIG2 is a crucial protein of HIF-1 $\alpha$-TGF-Snail/Smad, which related to cell immigration and epithelialmesenchymal transformation (EMT) result in metastasis under hypoxia [16]. From these reasons, to suppress this signal pathway seems to be one of important strategy for rapid metastatic tumors like clear cell carcinoma (CCC) of kidney [24], ovary [25].

We used the epitope peptides of its coding gene as antigen restricted HLA-A*0201 specifically. Unfortunately, we could not harvest epHIG2 restricted HLA*A-A24102.

FOXM1 is a key protein usually exist in cytoplasm of tumor cell ubiquitously and regulate cell cycle in $G 2 / M$ interacting with B-Myb (myeloblastosis family of transcription factors protein B), and Cyclin D-CDK4/6 and related to proliferation [26]. FOXM1 may be a promising target from this reason. Furthermore, FOXM1 progresses repairing of DNA [27], stemness [28], EMT [29], and resistance to paclitaxel+carboplatin [30]. In both OC and $\mathrm{CC}$, FOXM1 is strongly related to proliferation, invasion, metastases and angiogenesis via interacting to hedgehog pathway [31] or PIK3-mTOR pathways [30], as well. As for subtypes of OC, overexpression of FOXM1 was detected not only in high grade serous (HG-S) but also in clear cell carcinoma, endometrioid and mucinous carcinoma of ovary [32].

From these reasons, epFOXM1 was thought to be very promising and strikingly appropriate as selection.

MELK had been previously implicated as an oncogenic kinase. Its oncogenesis was identified by EZH2 (Enhancer of zeste homolog 2) pathway, and its proliferation was activated by NF-kB [33] pathway and EMT via TGF-beta-SMD signaling pathway [34].

MELK has also been shown to be important for mitosis and it regulates cell cycling G2/M collaborating with FOXM1 [35] and depresses P21 and inhibits apoptosis. Furthermore, the stemness [36] via STAT3and C-Jun [19] is enhanced by MELK as well, which may cause resistant to irradiation. Furthermore, recent study elucidated MELK is key peptides in poor prognostic OC (esp. HG-S with refractory disease) and activate the upstream of FOXM1 in proliferation and G2/M checkpoint inhibitor in cycling tumor cell and decrease apoptosis in such poor prognostic HG-S [35]. HJURP is a chaperone of CENP-A (centromere proteinA) which is necessary to mitosis. HJURP introduces the dimerization of CENP-A, and segregate to centromere, finally, CENP-A dimers must be tetra-dimer (octamer) in mitosis. HJURP inhibition is related to cell immortality via ataxia teleangiectasia receptor (ATR)- double strands breakage (DBS) stop and many kinds of fusion-genes cause genomic instability and carcinogenesis or uncontrollable proliferations via non-homologous end joining repairing (NHEJ) [22]. 
As for microenvironments of tumor, as well, HJURP depletion causes premature senescence in fibroblasts and endothelial cells, here, HJURP inhibition showed anti-tumor effect in direct manner via anti-angiogenesis. From these reason, HJURP gene derived epitope peptides seemed to very good target with dual (tumor cell and its microenvironmental cell) anti-cancer effects. Furthermore, the salvage angiogenesis would be enhanced due to hypoxia around the tumor cells by chemotherapy. Fibroblasts, peripheral macrophages, platelets and endothelial cells were activated by cross-talks with tumor cell via cytokines such as VEGFs, placental induced growth factor (PIGF), basic fibroblast growth factor (bFGF), platelet derived growth factors (PDGFs), interleukin 6, interleukin 8, interleukin 12, and so on. Considering those tumor-survival mechanisms, both antitumor and anti-microenvironment therapy would be necessary to eliminate the tumor cells.

In phase 1 enrollees, there were few of SCC and the adherence to URLC10 was low as $50 \%$. Usual frequency of subtypes in newly diagnosed uterine cancer showed that SCC covers about $70 \%$ of uterine CC in Japan. In our studies, concerning the hematological adverse events, there was none which related to agents. Grading of adverse events was assessed in comparison with base line. Recently, Hasegawa et al. conducted another phase 1 study of the same oncoantigens in small patients and reported its safety and tolerability but no response. He reported anemia in $77.8 \%$ (7/9), but we did not detect anemia due to PV even in CC. They reported hematological adverse event of anemia but most of them related to the property of the disease progression and not stated as vaccine related. Dermatologic reactions were only adverse event related to vaccine therapy like us, as well [37]. They may report pure events of AEs without discriminated assessment of disease induced or agent induced. We assessed every parameter of hematology in individual clinical course using difference of grading, and its causes were decided as due to agents, or disease progression, or patient's own character or unknown reason by comparing with patient's complaint and doctor's observation and consider. Dermatological reaction is major adverse events. Shown in table 6, the chief toxicity of this treatment was dermatologic reactions alone. They were feasible to continue of this treatment. No dose limiting toxicities were detected in this phase 1 part.

Response of $P V$

Shown in figures 3 and 4, tumor response was detected in $18 \%(2 \mathrm{PR} / 11)$ in $\mathrm{CC}$ and $25 \%(1 \mathrm{CR}+2 \mathrm{PR} / 12)$ in $\mathrm{OC}$. Longer survival was obtained in some patients, it is effective to maintain QOL and longer survival (QALY, both were the endpoints of P2). Considering the most of all these patients were announced as best supportive care, this peptides vaccine therapy gives the power and hope to live as they are in their social and familial wellbeing. As for this item, we are going to discuss in P2 part, later.

Immunological discovery

From the result of positivity in ELISpot assay, HJURP was smaller and weaker in positive rate. The reason was unclear and HJURP would not be a major key factor to proliferation of chemo-resistant patients or tumors of such patients were very slow growth but steady growth, so called smoldering disease.

As for the results of ELISpot assay, nine out of 22 (41\%) of patients had CTL active form to the antigens before this protocol treatment. 27\% (3/11) in CC and 55\% (6/11) in OC showed CTL activity before vaccination. Radiotherapy (esp. hypo-fraction) enhances the antigenicity in tumor but in this small case cohort its activity has not demonstrated [38]. Among the antigens showed positive before vaccination, MELK and HJURP in CC were prominent. On the other hand, VEGFR1 showed highly positive in OC. From these facts, it is speculated that salvage proliferation pathway became active in CC and VEGF may be key factor in OC. This fact would support 'Beyond PD effect' in OC like colon cancer [39]. In summary, prior treatment using chemotherapy or radiotherapy breaks the tumor cells and would disseminate various tumor cell antigens into serum. From these reasons, possibility of using these peptide-vaccine therapy as adjuvant treatment, at least one prior treatment of chemotherapy or radiotherapy would be necessary.

\section{Conclusion}

We conducted the immune-therapy using HLA-restricted tumor derived epitope-peptides vaccine to eliminate the chemo-resistant tumor cell in metastatic or recurrent focuses for OC and CC.

We had selected the peptides from crucial targets of salvage driver genes in proliferation (URLC10, FOXM1, MELK), in metastasis (HIG2) and in stemness (HJURP). The tumor cells had presented their epitopes on their cell membrane by $\mathrm{MCH} 1$ (HLA). So, they were good targets for activated CTLs by our vaccination, and the CTLs attacked the tumor cell 'by homing' and would eliminate the tumor cell alone.

Furthermore, from the tumor microenvironmental view, the vascular endothelial cells which had been calling for by tumor cell via VEGFR1, and R2 receptors (the ligands would be VEGFA produced by tumor cells) expressed the epVEGFR1 and R2 on the membrane of endothelial cell, and the CTLs activated by our vaccination using epVEGFR1 and R2 had eliminated the vascular endothelium cells and blocked new angiogenesis. The dual attacks to tumor cell and its neo vessels may be stunning strategy for conquering the cancer.

In conclusion, this presented multiple peptides cocktail vaccination therapy at this dose was very tolerable and effective. Based on these results further P2 part was continued to accrual.

These peptides vaccine cocktails therapies were safe and effective in these cohorts of patients.

\section{Acknowledgements}

We thank for co-operation of the institute of medical science, The university of Tokyo, in ELISPOT assay and delivery for all peptides in GMP grade. As for submission, 
we thank very much for Dr. Peter G Rose, Cleveland Clinic Main Campus, Gynecologic Oncology in the review of this manuscript concerning English.

\section{References}

[1] Hodi FS, O'Day SJ, McDermott DF, Weber RW, Sosman JA, Haanen JB, Gonzalez R, Robert C, Schadendorf D, Hassel JC, Akerley W, van den Eertwegh AJ, Lutzky J, Lorigan P, Vaubel JM, Linette GP, Hogg D, Ottensmeier CH, Lebbé C, Peschel C, Quirt I, Clark JI, Wolchok JD, Weber JS, Tian J, Yellin MJ, Nichol GM, Hoos A, Urba WJ (2010). Improved survival with ipilimumab in patients with metastatic melanoma. N Engl J Med. 19 (363): 711-723.

[2] Coleman R, Schachter J, Long GV, Arance A, Grob JJ, Mortier L, Daud A, Calino MS, McNeil C, Lotem M, Larkin J, Lorigan P, Neyns B, Blank CU, Hamid O, Mateus C, Shapira-Frommer R, Kosh M, Zhou H, Ibrahim N, Ebbinghaus S, Ribas A; KEYNOTE-006 investigators (2015). Pembrolizumab versus Ipilimumab in Advanced Melanoma. $\mathrm{N}$ Engl J Med 372 (26): 2521-2532. DOI: 10.1056/NEJMoa1503093. Epub 2015 Apr 19.

[3] Brahmer JR, Tykody SS, Chow LQ, Hwu WJ, Topalian SL, Hwu P, Drake CG, Camacho LH, Kauh J, Odunsi K, Pitot HC, Hamid O, Bhatia S, Martins R, Eaton K, Chen S, Salay TM, Alaparthy S, Grosso JF, Korman AJ, Parker SM, Agrawal S, Goldberg SM, Pardoll DM, Wigginton J (2012). Safety and activity of anti-PD-L1 antibody in patients with advanced cancer. N Engl J Med. 366 (26): 2455-2465. DOI: 10.1056/NEJMoa1200694.

[4] Mittica G, Ghisoni E., Giannone G, Aglietta M, Genta S, Valabrega G (2017). Checkpoint inhibitors in endometrial cancer: preclinical rationale and clinical activity. Oncotarget. 8 (52): 90532-90544. DOI: 10.18632/oncotarget. 20042.

[5] Suda T, Tsunoda T, Daigo Y, Nakamura Y, Tahara H (2007). Identification of human leukocyte antigen-A24-restricted epitope peptides derived from gene products upregulated in lung and esophageal cancers as novel targets for immunotherapy. Cancer Sci. 98 (11): 1803-1808. DOI: 10.1111/j.1349-7006.2007.00603.x.

[6] Yoshitake Y, Nishimura Y, Nakamura Y, Shinohara M (2015). A clinical trial of multiple peptides vaccination for advanced head and neck cancer patients induced immune responses and prolonged OS. Oncoimmunology. 1 (4): e1022307.ecollection PMID: 26405579 PMCID: PMC4570101 DOI: 10.1080/2162402X.2015.1022307

[7] Imai K, Hirata S, Irie A, Senju S, Ikuta Y, Yokomine K, Harao $M$, Inoue $M$, Tsunoda $T$, Nakatsuru $S$, Nakagawa $H$, Nakamura Y, Baba H, Nishimura Y (2008). Identification of a novel tumor-associated antigen, cadherin $3 / \mathrm{P}$-cadherin, as a possible target for immunotherapy of pancreatic, gastric, and colorectal cancers. Clin Cancer Res. 14 (20): 6487-6495. DOI: 10.1158/1078-0432.CCR-08-1086.

[8] Kono K, Mizukami Y, Daigo Y, Takano A, Masuda K, Yoshida K, Tsunoda T, Kawaguchi Y, Nakamura Y, Fujii H (2009). Vaccination with multiple peptides derived from novel cancer-testis antigens can induce specific T-cell responses and clinical responses in advanced esophageal cancer. Cancer Sci. 100 (8): 1502-1509. DOI: 10.1111/j.1349-7006.2009.01200.x.

[9] Siegel RL, Miller KD, Jemal A (2018). Cancer Statistics,
2018. CA CANCER J CLIN. 2018; 68: 7-30. DOI: $10.3322 /$ caac. 21442 .

https://onlinelbrary.wiley.com/doi/full/10.3322/caac.21442.

[10] Ozols RF, Bundy BN, Greer BE, Fowler JM, Clarke-Pearson D, Burger RA, Mannel RS, DeGeest K, Hartenbach EM, Baergen R; Gynecologic Oncology Group (2003). Phase III trial of carboplatin and paclitaxel compared with cisplatin and paclitaxel in patients with optimally resected stage III ovarian cancer: a Gynecologic Oncology Group study. J Clin Oncol. 21 (17): 3194-3200. DOI: 10.1200/JCO.2003.02.153.

[11] Katsumata N, Yasuda M, Takahashi F, Isonishi S, Jobo T, Aoki D, Tsuda H, Sugiyama T, Kodama S, Kimura E, Ochiai K, Noda K; Japanese Gynecologic Oncology Group (2009). Dose-dense paclitaxel once a week in combination with carboplatin every 3 weeks for advanced ovarian cancer: a phase 3, open-label, randomized controlled trial. Lancet. 374 (9698): 331-338. DOI: 10.1016/S0140-6736 (09)61157-0.

[12] Burger RA, Brady MF, Rhee J, Sovak MA, Kong G, Nguyen HP, Bookman MA (2013). Independent radiologic review of the Gynecologic Oncology Group Study 0218, a phase III trial of bevacizumab in the primary treatment of advanced epithelial ovarian, primary peritoneal, or fallopian tube cancer. Gynecol Oncol. 131 (1): 21-26. DOI: 10.1016/j.ygyno.2013.07.100.

[13] Tewari KS, Sill MW, Penson RT, Huang H, Ramondetta LM, Landrum LM, Oaknin A, Reid TJ, Leitao MM, Michael HE, DiSaia PJ, Copeland LJ, Creasman WT, Stehman FB, Brady MF, Burger RA, Thigpen JT, Birrer MJ, Waggoner SE, Moore DH, Look KY, Koh WJ, Monk BJ (2017). Bevacizumab for advanced cervical cancer: final overall survival and adverse event analysis of a randomized, controlled, open-label, phase 3 trial (Gynecologic Oncology Group 240). Lancet. 390 (10103): 1654-1663.

[14] Pujade-Lauraine E, Lederman JE, Selle F, Gebski V, Penson RT, Oza AM, Korach J, Huzarski T, Poveda A, Pignata S, Friedlander M, Colombo N, Harter P, Fujiwara K, RayCoquard I, Banerjee S, Liu J, Lowe ES, Bloomfield R, Pautier P; SOLO2/ENGOT-Ov21 investigators (2017). Olaparib tablets as maintenance therapy in patients with platinumsensitive, relapsed ovarian cancer and a BRCA1/2 mutation (SOLO2/ENGOT-Ov21): a double-blind, randomized, placebo-controlled, phase 3 trial. Lancet Oncol. (9): 12741284. DOI: 10.1016/S1470-2045 (17)30469-2.

[15] Isobe A, Sawada K, Kinose Y, Ohyagi-Hara C, Nakatsuka E, Makino H, Ogura T, Mizuno T, Suzuki N, Morii E, Nakamura K, Sawada I, Toda A, Hashimoto K, Mabuchi S, Ohta T, Morishige K, Kurachi H, Kimura T (2015). Interleukin 6 receptor is an independent prognostic factor and a potential therapeutic target of ovarian cancer. PLoS One. 10 (2): e0118080. DOI: 10.1371/journal.pone.0118080. eCollection 2015.

[16] Togashi A, Katagiri T, Ashida S, Fujioka T, Maruyama O, Wakumoto Y, Sakamoto Y, Fujime M, Kawachi Y, Shuin T, Nakamura Y (2005). Hypoxia-inducible protein 2 (HIG2), a novel diagnostic marker for renal cell carcinoma and potential target for molecular therapy. Cancer Res, 2005; 65: 48174826. DOI: 10.1158/0008-5472.CAN-05-0120.

[17] Ishizaki H, Tsunoda T, Wada S, Yamauchi M, Shibuya M, Tahara H (2006). Inhibition of tumor growth with antiangiogenic cancer vaccine using epitope peptides derived from human vascular endothelial growth factor receptor 1 . Clin Cancer Res. 12 (19): 5841-5849. DOI: 10.1158/10780432.CCR-06-0750. 
[18] Wada S, Tsunoda T., Baba T, Primus FJ, Kuwano H, Shibuya M, Tahara, H (2005). Rationale for antiangiogenic cancer therapy with vaccination using epitope peptides derived from human vascular endothelial growth factor receptor 2 . Cancer Res. 65: 4939-4946. DOI: 10.1158/0008-5472.can-04-3759.

[19] Yokomine K, Senju S, Nakatsura T, Irie A, Hayashida Y, Ikuta Y, Harao M, Imai K, Baba H, Iwase H, Nomori H, Takahashi K, Daigo Y, Tsunoda T, Nakamura Y, Sasaki Y, Nishimura Y (2010). The forkhead box M1 transcription factor as a candidate of target for anti-cancer immunotherapy. Int $\mathbf{J}$ Cancer. 2010; 123 (9): 2153-2163. DOI: 10.1002/ijc.24836.

[20] Gu C, Banasavadi-Siddegowda YK, Joshi K, Nakamura Y, Kurt H, Gupta S, Nakano I (2013). Tumor-specific activation of the C-JUN/MELK pathway regulates glioma stem cell growth in a p53-dependent manner. Stem Cells. 31 (5): 870881. DOI: $10.1002 /$ stem. 1322 .

[21] Seo T, Konda R, Sugimura J, Iwasaki K, Nakamura Y, Fujioka $\mathrm{T}$ (2010). Expression of hypoxia-inducible protein 2 in renal cell carcinoma: A promising candidate for molecular targeting therapy. Oncol Lett. 1 (4): 697-701. DOI: 10.3892/ol 00000122 .

[22] Kanehira M, Katagiri T, Shimo A, Takata R, Shuin T, Miki T, Fujioka T, Nakamura Y (2007). Oncogenic role of MPHOSPH1, a cancer-testis antigen specific to human bladder cancer. Cancer Res. 67 (7): 3276-3285. DOI: 10.1158/0008-5472.CAN-06-3748.

[23] Mizukami Y, Kono K, Daigo Y, Takano A, Tsunoda T, Kawaguchi Y, Nakamura Y, Fujii H (2008). Detection of novel cancer-testis antigen-specific T-cell responses in TIL, regional lymph nodes, and PBL in patients with esophageal squamous cell carcinoma. Cancer Sci. 99: 1448-1454. DOI: 10.1111/j.1349-7006.2008.00844.x.

[24] Fujiwara Y, Sugimura K., Miyata H, Omori T, Nakano H, Mochizuki C, Shimizu K, Saito H, Ashida K, Honjyo S, Nakamura Y, Yano M (2017) A Pilot Study of Post-Operative Adjuvant Vaccine for Advanced Gastric Cancer. Yonago Acta Med. 60 (2): 101-105. PMCID: PMC5502221.

[25] Obara W, Karashima T, Takeda K, Kato R, Kato Y, Kanehira M, Takata R, Inoue K, Katagiri T, Shuin T, Nakamura Y, Fujioka T (2017) Effective induction of cytotoxic $\mathrm{T}$ cells recognizing an epitope peptide derived from hypoxiainducible protein 2 (HIG2) in patients with metastatic renal cell carcinoma. Cancer Immunol Immunother. 66 (1): 17-24. DOI: $10.1007 / \mathrm{s} 00262-016-1915-5$.

[26] Nishimura S, Tsuda H., Ito K, Takano M, Terai Y, Jobo T, Kigawa J, Sugiyama T, Yaegashi N, Aoki D (2010). Differential expression of hypoxia-inducible protein 2 among different histological types of epithelial ovarian cancer and in clear cell adenocarcinomas. Int J Gynecol Cancer. 20: 220226. DOI: 10.1111/IGC.0b013e3181ca1e16.

[27] Down CF, Millour J, Lam EW, Watson RJ (2012). Binding of FoxM1 to G2/M gene promoters is dependent upon B-Myb. Biochim Biophys Acta. 1819 (8): 855-862. DOI: 10.1016/j.bbagrm.2012.03.008.

[28] Millour J, de Olano N, Horimoto Y, Monteiro LJ, Langer JK, Aligue R, Hajji N, Lam EW (2011). ATM and p53 regulate FOXM1 expression via E2F in breast cancer epirubicin treatment and resistance. Mol Cancer Ther. 10 (6): 1046-1058. DOI: 10.1158/1535-7163.MCT-11-0024.
[29] Xu XS, Miao RC, Wan Y, Zhang LQ, Qu K, Liu C (2015). FoxM1 as a novel therapeutic target for cancer drug therapy. Asian Pac J Cancer Prev. 2015; 16 (1): 23-29. 10.7314/apjcp. 16.1.23.

[30] Roh YG, Mun MH, Jeong MS, Kim WT, Lee SR, Chung JW, Kim SI, Kim TN, Nam JK, Leem SH (2018). Drug resistance of bladder cancer cells through activation of ABCG2 by FOXM1. BMB Rep., $51 \quad$ (2): 98-103. DOI: 10.5483/bmbrep.2018.51.2.222.

[31] Chen H, Zou Y, Yang H, Wang J, Pan H (2014). Downregulation of FoxM1 inhibits proliferation, invasion and angiogenesis of HeLa cells in vitro and in vivo. Int $\mathrm{J}$ Oncol. 45 (6): 2355-64. DOI: 10.3892/ol.2016.4932.

[32] Barger CJ, Zhang W, Hillman J, Stablewski AB, Higgins MJ, Vanderhyden BC, Odunsi K, Karpf AR (2015). Genetic determinants of FOXM1 overexpression in epithelial ovarian cancer and functional contribution to cell cycle progression. Oncotargets. 6 (29): $27613-27627 . \quad$ DOI: 10.1016/j.ygyno.2013.07.100.

[33] Wang Y, Lee YM, Baitsch L, Huang A, Xiang Y, Tong H, Lako A, Von T, Choi C, Lim E, Min J, Li L, Stegmeier F, Schlegel R, Eck MJ, Gray NS, Mitchison TJ, Zhao JJ (2014) MELK is an oncogenic kinase essential for mitotic progression in basal-like breast cancer cells. Elife, 3: e01763. DOI: $10.7554 /$ eLife. 01763.

[34] Janostiak R, Rauniyar N, Lam TT, Ou J, Zhu LJ, Green MR, Wajapeyee N (2017). MELK Promotes Melanoma Growth by Stimulating the NF-кB Pathway. Cell Rep. 2017; 21 (10): 2829-2841. DOI: 10.1016/j.celrep.2017.11.033.

[35] Cheng J, Qin B, Liu B, Huang T, Li Y, Ma L (2017). Maternal embryonic leucine zipper kinase inhibits epithelialmesenchymal transition by regulating transforming growth factor- $\beta$ signaling. Oncol Lett. 13 (6): 4794-4798. DOI: 10.3892/ol.2017.6081

[36] Kohler RS, Kettlehack H, Knipprath-Mészaros AM, Fedier A, Schoetzau A, Jacob F, Heinzelmann-Schwarz V (2017). MELK expression in ovarian cancer correlates with poor outcome and its inhibition by OTSSP167 abrogates proliferation and viability of ovarian cancer cells. 145 (1): 159-166. DOI: 10.1016/j.ygyno.2017.02.016.

[37] Hasegawa K, Ikeda Y, Kunugi Y, Kurosaki A, Imai Y, Kohyama S, Nagao S, Kozawa E, Yoshida K, Tsunoda T, Nakamura Y, Fujiwara K (2018). Phase I Study of Multiple Epitope Peptide Vaccination in Patients with Recurrent or Persistent Cervical Cancer. J Immunother., 41 (4): 201-207. DOI: 10.1097/CJI.0000000000000214.

[38] Campbell AM, Decker RH. (2017) Mini-review of conventional and hypofractionated radiation therapy combined with immunotherapy for non-small cell lung cancer. Transl Lung Cancer Res. 6 (2): 220-229. DOI: 10.21037/tlcr.2017.03.02.

[39] Bennouna J, Sastre J, Arnold D, Österlund P, Greil R, Van Cutsem E, von Moos R, Viéitez JM, Bouché O, Borg C, Steffens CC, Alonso-Orduña V, Schlichting C, Reyes-Rivera I, Bendahmane B, André T, Kubicka S; ML18147 Study Investigators (2013). Continuation of bevacizumab after first progression in metastatic colorectal cancer (ML18147): a randomized phase 3 trial. Lancet Oncol. 14 (1): 29-37. DOI: 10.1016/S1470-2045(12)70477-1. 